\title{
Introduction to polar earth orbits (PEO) in the function of the satellite distress and safety systems (SDSS)
}

\author{
Dimov Stojce Ilcev \\ Space Science Centre (SSC), Durban University of Technogy (DUT), South Africa
}

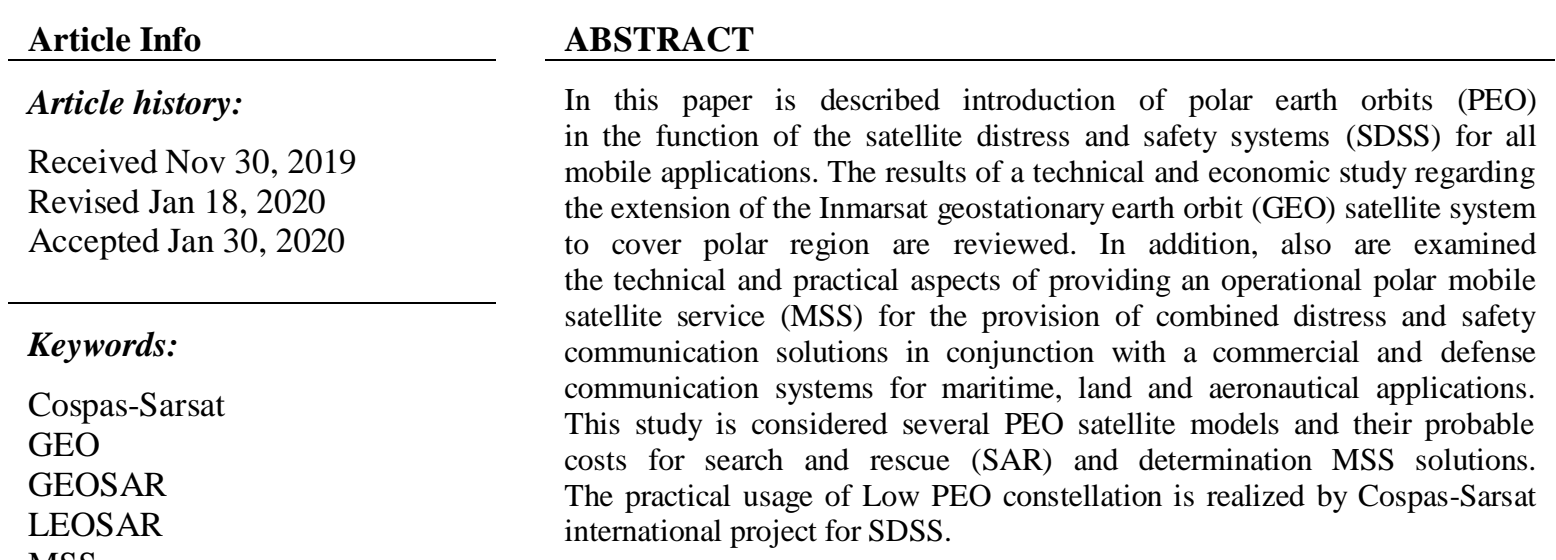

MSS

Polar Earth Orbits (PEO)

SDSS

Copyright $\odot 2020$ Institute of Advanced Engineering and Science. All rights reserved.

\section{Corresponding Author:}

Dimov Stojce Ilcev,

Space Science Centre (SSC),

Durban University of Technogy (DUT),

Durban, South Africa.

168 University Road, Negara.

Email: ilcev@dut.ac.za

\section{INTRODUCTION}

The polar earth orbits (PEO) satellite constellation is today a synonym for providing coverage of both Polar Regions for emergency, different meteorological observation and satellite determination services. A satellite in this orbit travels its course over both geographical poles and effectively follows a line of longitude. Certainly, this orbit may be virtually circular or elliptical depending upon requirements of the program and is inclined at about $90^{\circ}$ to the equatorial plane, covering both poles.

The orbit is fixed in space while the Earth rotates underneath and consequently, the satellite, over a number of orbits determined by its specific orbit line, will pass over any given point on the Earth's surface. A single satellite in a PEO plane provides in principle coverage to the entire globe, although there are long periods during which the satellite is "out of view" of a particular ground earth station (GES). Accessibility can of course be improved by deploying more than one satellite in different orbital planes. Thus, if two such satellite orbits are spaced at $90^{\circ}$ to each other, the time between their passes over any given point will be halved.

The PEO system is rarely used for communication purposes because this satellite is in real view of a specific point on the Earth's surface for only a short period of time. In addition, any complex steerable antenna systems would also need to follow the satellite as it passes overhead. At any rate, this special satellite orbit may be acceptable for a processing store-and-forward type of low data mobile communications system and for satellite determination and navigation [1-3].

There are four primary requirements for PEO systems as: 
a) To provide global satellite visibility, coverage and access links for existing Low Earth Orbit (LEO) LEOSAR SDSS system of Cospas-Sarsat distress and safety radio beacons such as: Maritime EPIRB (Emergency Position Indicating Radio Beacon), Land Vehicle/Personal PLB (Personal Locator Beacon) and Aeronautical ELT (Emergency Locator Transmitter);

b) To provide global continuous satellite coverage for current or newly developed and forthcoming satellite navigation systems;

c) To provide communication requirements at RF L-band or any convenient spectrum for ships and aircraft in the polar regions not covered by the Inmarsat system; and

d) To provide global coverage for meteorological and synoptic observation stations.

The Inmarsat expert's staff has studied two PEO solution for distress and communication purposes, first, low altitudes up to $1,400 \mathrm{~km}$ and second, high altitudes above $11,000 \mathrm{~km}$. In fact, these two orbit PEO ranges are separated by the Inner Van Allen radiation belt. In the regions of the radiation belt the radiation level increases roughly exponentially with height at around $1,000 \mathrm{~km}$, reaching a peak at about $5,000 \mathrm{~km}$ altitude. A critical requirement to reduce high-energy proton damage to the solar cell arrays of the satellite system constrains the PEO to low and high orbital altitudes. As is evident, another Outer Van Allen Belt has no so negative influence on these two PEO satellite constellations, because this belt lies far a way between medium earth orbit (MEO) and GEO satellite planes.

These two specific systems studied by Inmarsat are Cospas-Sarsat Low PEO at 1,000 km altitude and High PEO at 12,000 km altitude, similar to that studied by ERNO, named SERES (Search and Rescue Satellite) system. In such a way, it is considered that these two satellite orbital systems demonstrate clearly the solutions tradeoff and constraints on a joint PEO distress, safety, SAR and communication mission.

Other possible orbits for total polar coverage can be an inclined Highly Elliptical Orbit (HEO) of Russian Molnya constellation of 4 satellites; GIO (Geosynchronous Inclined Orbit) at $45^{\circ}$ inclined plane of 4 satellites and $55^{\circ}$ inclined circular MEO at 20,000 km altitude for both the US GPS and Russian GLONASS military Global Satellite Navigation Systems (GNSS) [4-8].

\section{COSPAS-SARSAT LEOSAR SYSTEM}

The Cospas-Sarsat LEOSAR system is SDSS network that uses PEO satellites of non-continuous coverage in LEO orbit provided by special LEOSAR configuration. Thus, the use of LEO low-altitude orbiting satellites provides a strong Doppler effect in the up-link signal, which allows the use of Doppler positioning techniques for special satellite beacons. The distress signals of SDSS network radiated by these beacons, such as EPIRB for oceangoing ships, PLB for persons and land vehicles and ELT for all kind of aircraft, at $406 \mathrm{MHz}$ are received by a first or second generation of LEO/PEO satellites via Local User Terminals (LUT) of LEOLUT stations equipped with suitable transponders and antenna systems. These detected distress signals are relayed by LEOSAR satellites on $1544.5 \mathrm{MHz}$ a corresponding LEOLUT station, where the signals are processed to determine the location of the satellite beacons and/or a mobile in distress.

Doppler positioning is using the relative motion between the spacecraft and the LEOSAR beacons has been chosen as the only practical means of locating distress signals. The carrier RF radiated by the beacon is reasonably stable during the period of mutual beacon and satellite visibility. In the past Cospas-Sarsat used the VHF band of $121.5 \mathrm{MHz}$ (international aeronautical emergency frequency) and today is using UHF band of 406.0 to $406.1 \mathrm{MHz}$ exclusively reserved for distress beacons operating with satellite systems.

Although the UHF beacons are more sophisticated than the VHF beacons, through the addition of identification and situation codes in the messages, complexity is still kept to a minimum by the retention of the Doppler location concept. Another VHF band on $243 \mathrm{MHz}$ is usually only in service for military and not in Sarsat distress communications any more. To optimize Doppler location, a LEO near polar orbital plane is used. The Doppler effect is an event causing frequency changes of the received radio signals for a time during the relative motion of the radio beacon transmitters and/or satellite receivers:

$$
\mathrm{fD}=\mathrm{f}^{\prime} \mathrm{V}^{\prime} / \mathrm{c}=2\left(\mathrm{f}^{\prime} \mathrm{V} \cos \alpha / \mathrm{c}\right)=\mathrm{V} \cos \alpha / \lambda[\mathrm{Hz}]
$$

Where $1 / \lambda=\mathrm{f}^{\prime} / \mathrm{c}, \mathrm{f}^{\prime}$ is the frequency of the electromagnetic waves, $\alpha$ is the angle between real relative speed $(\mathrm{V})$ and radial relative speed (V') shared by Tx and Rx. The Doppler frequency (fD) is directly proportional to the relative speed of Tx and Rx and inversely proportional to the wavelength.

The Doppler effect is not all theoretical though, which equation can be used to calculate both the velocity of the source and observer, the original frequency of the sound waves and the observed frequency of the sound waves. The observer frequency $\left(\mathrm{f}_{\mathrm{o}}\right)$ can be calculated with the following equation: 


$$
f_{o}=\left[\left(V+V_{o} / V-V_{s}\right)\right] f
$$

Where $\mathrm{f}$ - actual frequency, $\mathrm{V}$ - velocity of sound waves, $\mathrm{V}_{\mathrm{o}}=$ velocity of the observer, and $\mathrm{V}_{\mathrm{s}}=$ velocity of source. While there is only one Doppler effect relation, the equation changes in different situations depending on the velocities of the observer (object) or the source of the sound. At this point, the Doppler effect equation can be applied in different situations [3, 9-11].

\subsection{Source moving towards the observer or object at rest}

The immobile observer or object in this scenario is standing but another object is moving towards observer. More exactly, let's go back to (2). The velocity of the observer is zero, and so $\mathrm{V}_{\mathrm{o}}$ is equal to zero. Then, plugging this into the equation above, the new equation when a source is moving towards an observer at rest will be presented with the following relation:

$$
\mathrm{f}_{\mathrm{o}}=\mathrm{V} /\left(\mathrm{V}-\mathrm{V}_{\mathrm{s}}\right) \mathrm{f}
$$

\subsection{Source moving away from the observer or object at rest}

In this case one might imagine that some moving object passed next to the observer position at speed of about $25 \mathrm{~m} / \mathrm{s}$, which is still stationary. The frequency of the sound emitted by the moving object is $1,000 \mathrm{~Hz}$, and the sound waves travel with a velocity of $343 \mathrm{~m} / \mathrm{s}$. Since the observer velocity is zero, the value $\mathrm{V}_{\mathrm{o}}$ can be eliminate again from the equation. But this time, the moving object is going away from the observer, so its velocity is negative to indicate the direction. In such a way, the following equation for observer frequency will be as follows:

$$
f_{o}=V /\left[\left(V-\left(-V_{s}\right)\right] f\right.
$$

Inserting above values of numbers into the equation will provide the following result:

$$
\mathrm{f}_{\mathrm{o}}=343 \mathrm{~m} / \mathrm{s} /(343 \mathrm{~m} / \mathrm{s}+25 \mathrm{~m} / \mathrm{s}) 1000 \mathrm{~Hz}=932 \mathrm{~Hz}
$$

A source moving away from the observer creates a lower perceived frequency of sound waves, or according to the example, the frequency perceived by the observer is less than the actual frequency emitted by the siren, just what was expected from the Doppler effect [3].

\subsection{Observer Moving Toward a Stationary Source}

Now somebody can imagine that object is moving towards a loud block party. As somebody moves towards the speakers, the sound waves get closer together and the frequency increases. In this case, object is moving, but the source is not. So, value of $\mathrm{V}_{\mathrm{s}}$ will be equal to zero, so Doppler effect equation for an observer moving towards a stationary source will be presented with the following relation:

$$
\left.\mathrm{f}_{\mathrm{o}}=\left[\left(\mathrm{V}+\mathrm{V}_{\mathrm{o}}\right) / \mathrm{V}\right]-\left(-\mathrm{V}_{\mathrm{s}}\right)\right] \mathrm{f}
$$

\subsection{Observer moving away from a stationary source}

The moving object passed the loud party and they are on the way to a more serene setting. Now this object is moving away from the sound waves, the frequency decreases and velocity becomes and new equation is as follows:

$$
\left.f_{o}=\left[\left(V+V_{o}\right) / V\right]-\left(-V_{s}\right)\right] f
$$

Therefore, instead of adding $\mathrm{V}_{\mathrm{o}}$, we now subtract, since $\mathrm{V}_{\mathrm{o}}$ is negative.

Hence, when one of the LEOSAR satellites is approaching the position of beacon its Rx is detecting higher nominal RF rates radiated, when the satellite is just over the position of the beacon, both RF rates are identical and when the satellite is distant from the beacon, the received frequency becomes lower than the radiated one. This RF divergence is Doppler shift, which can be shown by the letter $\mathrm{S}$ curve form of the RF over time. The form and steep slope of the Doppler curve together with the position of the satellite on every point of the curve is used for determining the position of the beacon transmitter.

The Doppler location concept provides two positions for each beacon: the true position and its mirror image relative to the satellite ground track. This ambiguity is obviously resolved by calculations that take into account the Earth's rotation. If the beacon stability is good enough, as with $406 \mathrm{MHz}$ beacons, which are designed for this purpose, the true solution is determined over a single satellite pass. In the case 
of $121.5 \mathrm{MHz}$ beacons, the ambiguity is resolved by the result of the second satellite pass if the first attempt is unsuccessful. Location accuracy is also significantly better with $406 \mathrm{MHz}$ beacons shown in Table $1[3,12-15]$.

Table 1. Comparison of VHF and UHF satellite beacons

\begin{tabular}{lll}
\hline Characteristics & Ceased VHF 121.5 MHz (Ceased) & UHF 406 MHz \\
\hline Detection probability & (not applicable) & 0.88 \\
Location probability & 0.9 & 0.9 \\
Ambiguity resolution & $17.2 \mathrm{~km}$ & $90 \%$ within $5 \mathrm{~km}$ \\
Capacity & 10 & 90 \\
\hline
\end{tabular}

As depicted in Figure 1 (Left) a single PEO satellite circling the Earth around the poles, eventually views the entire Earth's surface. The "orbital plane", or path of the satellite, remains fixed, while the Earth rotates underneath. At most, it takes only one half rotation of the Earth (i.e., 12 hours) for any location to pass under the orbital plane. With a second satellite, having an orbital plane at right angles to the first, only one quarter of a rotation is required, or 6 hours maximum. Similarly, as more satellites orbit the Earth in different planes, the waiting time is further reduced. Thus, the COSPAS-SARSAT SDSS system design constellation is four PEO LEOSAR satellites, which provide a typical waiting time of less than one hour at mid-altitudes, shown in Figure 1 (Right). The altitude of the Cospas spacecraft orbit is approximately $1,000 \mathrm{~km}$, while that of Sarsat spacecraft is $850 \mathrm{~km}$. These low orbit altitudes result in a low uplink power requirement, a pronounced Doppler shift and short intervals between successive passes of spacecraft.
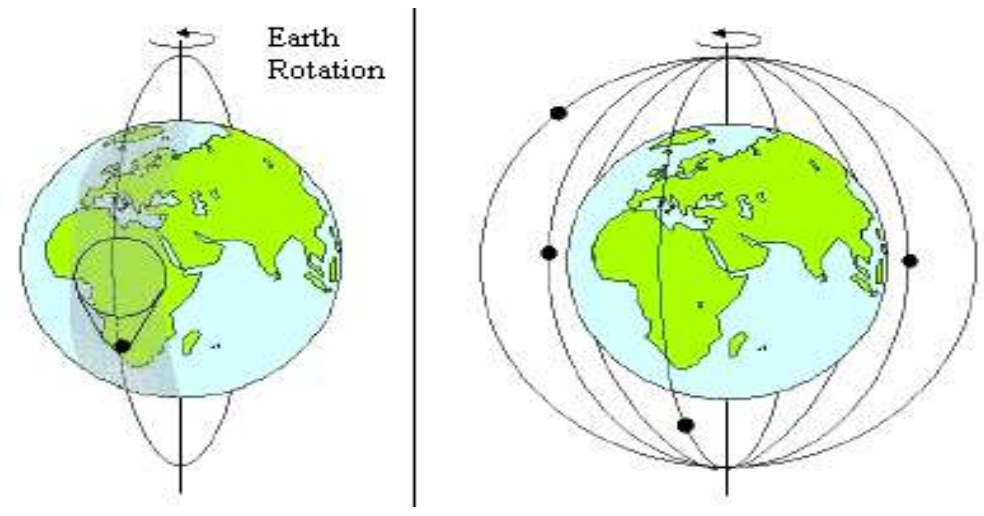

Figure 1. Diagrams of LEO satellites in near-PEO constellation

The near PEO could provide full global coverage but $121.5 \mathrm{MHz}$ alerts only if the relayed signals are received by a LUT. Namely, this constraint of the $121.5 \mathrm{MHz}$ system limits the useful coverage to the geographic area about 3,000 km radius around each LUT where the satellite can be simultaneously in the visibility of the transmitting beacon and the receiving LUT station. Thus, the improved performance of the $406 \mathrm{MHz}$ maritime EPIRB unit is the reason these small devices were selected by IMO for the GMDSS network and included in the 1988 amendments to the 1974 SOLAS Convention. Soon after the ICAO, in a similar way, recommended ELT beacons for avionics utilization.

In the meantime, the Cospas-Sarsat organization has developed a special GEO Search and Rescue (GEOSAR) system using three GEO satellites for global distress communications satellite beacons in combination with already developed LEO Search and Rescue (LEOSAR) systems using four Low Earth Orbit (LEO of PEO satellite constellations. For both the Low and High PEO systems the numbers of operational satellites are required to provide adequate Thus, Earth coverage needs to be quite minimized in order to achieve the minimum system costs. An IMO (International Maritime Organization) and ICAO (International Civil Aviation Organization) requirements for the Cospas-Sarsat mission is that there should be no time delay in distress alerting and call anywhere in the globe $[3,16,-19]$. 


\section{LOW PEO CONSTELLATION}

The Low PEO constellation similar to the Low Earth Orbits (LEO) mostly employs both polar and near-polar orbits for communications and navigation utilities. Thus, a particular example of a satellite system that uses this type of orbit is the Cospas-Sarsat SAR network for maritime, land and aeronautical applications. This system uses 8 satellites in 4 near-polar orbits: four US-based Sarsat Low PEO satellite constellations at $860 \mathrm{~km}$ orbits, inclined at $99^{\circ}$, which makes them sun-synchronous and four Russian Cospas satellite configurations at $1,000 \mathrm{~km}$ orbits, inclined at $82^{\circ}$. However, this orbit was also suitable for the first satellite navigation systems Transit and Cicada, developed by the USA and the former USSR (Russia) respectively. Otherwise, with a limited number of low altitude PEO satellites in LEO constellations it is impossible to provide reliable and continuous coverage to polar region, because the view of individual PEO spacecraft is relatively small and their transit time is short.

In Figure 2 is illustrated the Earth track for ten successive orbits of satellite in Low PEO with an altitude of about 1,000 km. The MES (Mobile Earth Station) in shaded area A (4,200 km in diameter) would see the satellite, in the absence of environmental screening, at an angle of elevation not less than $10^{\circ}$, while the satellite was passing through the equatorial plane. The coverage area has the same size and shape wherever the satellite is in the orbit but its apparent size and shape would change with latitude, being distorted by the map projection used in the Figure.

The South Pole coverage area at a single pass of the satellite is shown by shaded area $\mathrm{B}$. The same Figure shows that a single PEO satellite in a polar orbit will have a brief sighting of every part of the Earth's surface every day. There will be 2 or 3 of these glimpses per day near the equator, the number increasing as the poles approach. The period of visibility, as seen from the MES range and from about 10 minutes, the satellite passing overhead, down to a few seconds when the satellite appears briefly above the horizon.

However, because the time for a single orbit of PEO satellite is low, less then two hours and a different section of the polar region is covered at each orbit due to Earth rotation, this drawback is somewhat offset. For a given number of satellites, preferably about eight PEO, it is possible to optimize the constellation that maximizes total coverage, then to improve handover or minimize waiting time between transits.

As stated, the Cospas-Sarsat system of PEO satellites in LEO constellations is referred to as the Cospas-Sarsat LEOSAR System. Polar orbiting satellites used in the Cospas-Sarsat LEOSAR System can provide a global, but non-continuous coverage, for the detection and the positioning of distress beacons using a Doppler location technique. However, the non-continuous coverage introduces delays in the alerting process since the user in distress must "wait" for a satellite pass in visibility of his distress beacon. Thus, to eliminate these problems, Cospas-Sarsat system is using GEO and MEO satellite constellations.

If the PEO satellite orbital plane is given an angle of inclination differing from $90^{\circ}$, a similar Earth track is obtained but the geographical distribution of the satellite visibility changes. In such a way, one LEO satellite with an orbital inclination of $50^{\circ}$ would have better visibility between $60^{\circ} \mathrm{N}$ and $60^{\circ} \mathrm{S}$ latitude than a PEO satellite but it would have no visibility at all of the polar regions. The scenario of Low PEO satellite constellation, which sample with two satellites is shown in Figure 3 (Left), is attractive for mobile determination, tracking and distress communications for the following two reasons:

Firstly, the transmission path loss is relatively low, allowing reliable communication with a low powered satellite beacon and PEO spacecraft. An altitude of about 1,000 km is the upper limit for good reception of signals at 243/406 $\mathrm{MHz}$ sent from emergency distress beacons.

Secondly, the Doppler shift is high, approximately $30 \mathrm{kHz}$ at $1.6 \mathrm{GHz}$, allowing accurate location of the distress transmitter. On the other hand, there are several significant disadvantages.

However, as mentioned earlier, two satellites in PEO cannot provide continuous coverage unless there is simultaneous communication between a distress buoy and a ground terminal because of the small footprint of each individual satellite. Accordingly, message storage and retransmission of distress messages on-board processing would be necessarily adding to the distress alert delay time and also to satellite mass and complexity.

The short visibility period during a transit and the uneconomic need for large numbers of satellites for continuous network coverage makes Low PEO satellites unattractive for mobile communications considerations. If this orbit configured well as an economic solution for distress coverage in Polar Regions to be used for communications purposes, users would have to operate with the following four restrictions:

a) Only burst mode, low speed non-simultaneous data communication would be possible;

b) Transmission time and/or bit rate would be limited by satellite message storage capability;

c) Replies to the message would require an interrogation or polling system from the MES expecting a reply; and

d) Depending on the PEO constellation and MES position, a reply could take some hours. 
Many of these PEO satellite communication limitations would be removed if a system of inter-satellite links, possibly in addition to inter-GEO or HEO satellite infrastructures, were used to provide an extended near-continuous, and simultaneous two-way communication system. The complexity and likely cost of such system would almost certainly not be justified by the expected very low level of polar communication traffic. Accordingly, in considering the possible integration of PEO and GEO for communication purposes, it will necessary to determine the additional requirements and constraints arising from polar operation.

In this context, for reliable communications the number of additional LES (Land Earth Station) required for operation to PEO is a significant element of the overall system. For example, a constellation of eight Low PEO would require about six LES worldwide for polar coverage assuming data storing and forwarding techniques, where a High PEO would require a minimum of two LES located in North and South polar latitudes for continuous polar coverage with simultaneous two-way communications. This is not all, it would be necessary to obtain reliable terrestrial links between the LES of each system, as well as inter-satellite links between the PEO and GEO satellites.

In any case, by using the store and forward (transmit) method, a Low PEO system could effectively be served for the Cospas-Sarsat international project providing Satellite Distress and Safety Systems (SDSS). Thus, this GDSS system can relay of mobile distress, safety and urgency messages, for maritime, land and aeronautical applications via satellite beacons to receive-only terminals on shore [3, 20-23].

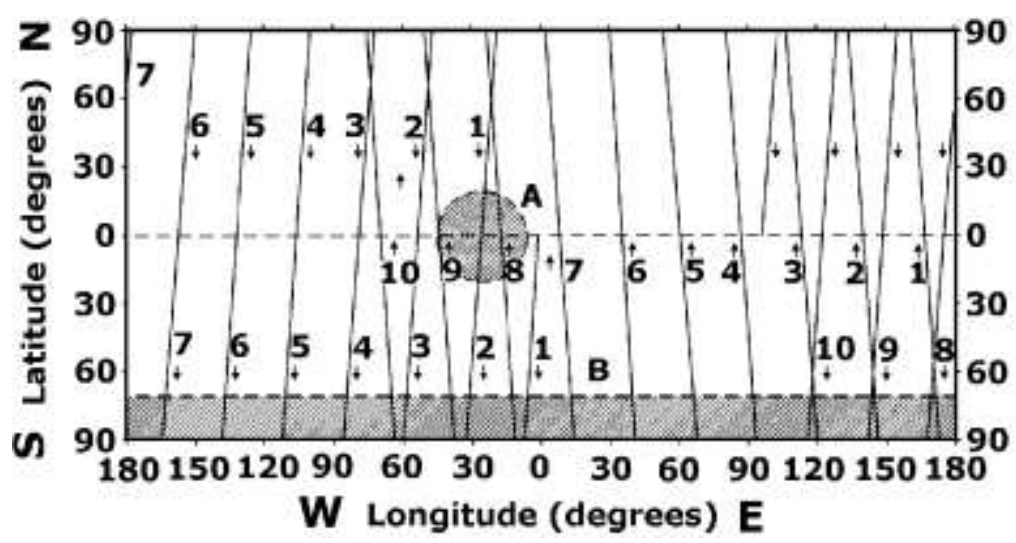

Figure 2. PEO Satellite Tracks
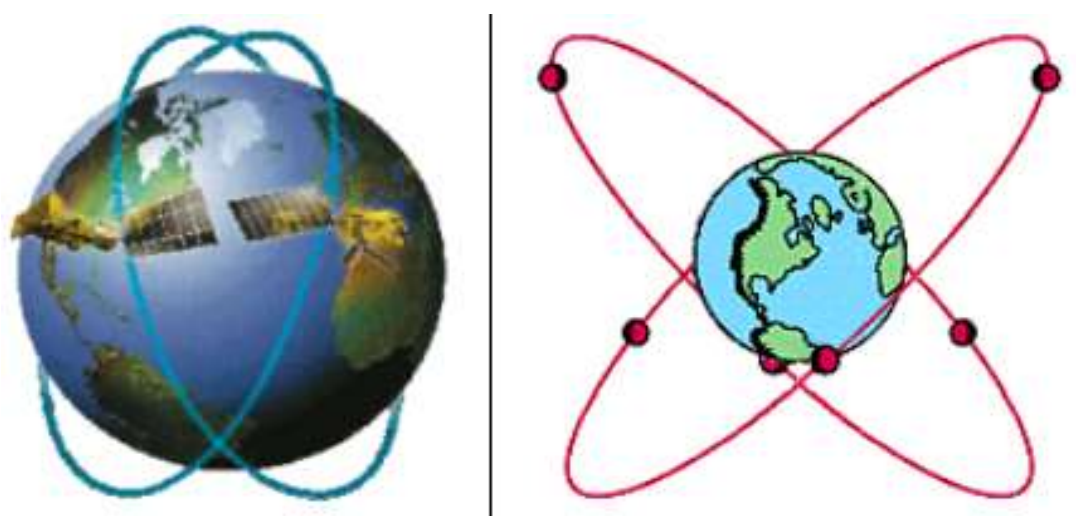

Figure 3. Low and high PEO satellite constellations

\section{HIGH PEO CONSTELLATION}

The High PEO constellation, shown in Figure 3 (Right), would consist of three satellites separated by $120^{\circ}$ in the same circular orbit of $12,000 \mathrm{~km}$ altitude, geometrically similar to the GEO and as orbit similar to MEO configuration. This orbit provides continuous coverage to all Polar Regions above $59^{\circ}$ latitude. 
At this point, six satellites (in two orbital planes of three satellites each) would provide continuous and real global coverage if that was required, which GEO cannot obtain. By comparison with Low PEO systems transmission path losses are higher at an altitude of 12,000 $\mathrm{km}$ but not to the extent that a distress beacon need be especially high powered to transmit successfully to a high PEO satellite. In fact, reception of the Cospas-Sarsat existing two very low-powered alert and distress frequencies will be quite interfered, but not impossible.

The Doppler shift is lower (about $10 \mathrm{kHz}$ at $1.6 \mathrm{GHz}$ ), not allowing very accurate area location of the distress transmitters. Single high latitude LES in both Arctic and Antarctic Polar Regions allows reception with no delay of all distress messages transmitted from above $59^{\circ}$ latitude. Using these two polar LES positioned at high latitude with continuous visibility of at least one of the three satellites and collocated or linked to the Inmarsat LES, can offer a full range of near continuous communications to the Polar Regions $[3,24,25]$.

\section{CONCLUSION}

The study that has been performed indicates that there are several top and modern technical and technology solutions to the problem of providing coverage of the Polar Regions for mobile communications, determination, surveillance, distress and safety applications. The need of reliable distress and safety solutions for maritime and aeronautical applications is obvious and the cost of network is insignificant, although the real communication requirements for these critical areas exist, they are difficult to quantify. The other existing HEO, MEO, LEO and their satellite orbits combinations can provide much better navigation, tracking and communication facilities for Polar Regions, so it is unlikely that the introduction of an PEO communication satellite system alone could ever be justified commercially. On the other hand, the practical and operational non-commercial Cospas-Sarsat PEO constellation is recently well established for SDSS, with its three LEOSAR, MEOSAR and GEOSAR subsystems. Low cost system providing a limited, reliable polar communication facility, in conjunction with an effective emergency, distress and safety capability and possibly other services may well be feasible if a means is found to finance and operate such PEO satellite systems.

\section{REFERENCES}

[1] Gallagher B., "Never Beyond Reach," Inmarsat, London, 1989.

[2] CNES, "Utilisation des satellites pour les recherches et le sauvetage," Cepadues, Toulouse, 1984.

[3] lcev D. S., "Global Mobile Satellite Communications for Maritime, Land and Aeronautical Applications,"Springer, vol. 1 Boston, 2016.

[4] Ilcev, D. S., "Global Mobile Satellite Distress and Safety Systems (SDSS)," Durban University of Technology (DUT), Durban, 2008.

[5] Ilcev D. S., "Global Mobile_Communications, Navigation and Surveillance (CNS),"Manual, Durban University of Technology (DUT), Durban 2014.

[6] S. D. Ilcev, "Hybrid satellite orbits (HSO) for global coverage," In 20th International Crimean Conference "Microwave \& Telecommunication Technology," pp. 404-405, Sevastopol, 2010.

[7] S. D. Ilcev, "Low Earth Orbits (LEO)," In 20th International Crimean Conference "Microwave \& Telecommunication Technology, pp. 406-408, Sevastopol, 2010.

[8] S. D. Ilcev, "Polar Earth Orbits (PEO)," In 20th International Crimean Conference "Microwave \& Telecommunication Technology, pp. 413-415, Sevastopol, 2010.

[9] Ilcev D. S., "Cospas-Sarsat LEO and GEO: Satellite Distress and Safety System (SDSS)," International Journal of Satellite Communications and Networking, vol. 25, no. 6, pp. 559-573, London, 2007.

[10] Richharia M., "Mobile Satellite Communications-Principes and Trends," Addison-Wesley, Harlow, 2001.

[11] Prentiss S., "Satellite communications", TAB, Blue Ridge Summit, 1987.

[12] ALRS, "Global Mobile Distress and Safety System (GMDSS)," Admiralty List of Radio Signals, vol. 5, UK, 2009.

[13] STUDY.COM, “Doppler Shift: Definition \& Formulas,” Working Scholars, 2019.

[14] I. Ali, N. Al-Dhahir and J. E. Hershey, "Doppler characterization for LEO satellites," in IEEE Transactions on Communications, vol. 46, no. 3, pp. 309-313, March 1998.

[15] Calcutt D. \& Tetley L., "Satellite Communications, Principles and Applications," Elsevier, Oxford, 2004.

[16] Guidotti A., Coralli V. A., Caus M., Bas J., Colavolpe G., Foggi T., Cioni S., \& Modenini A., "Satellite enabled LTE systems in LEO Constellations," IEEE International Conference on Communications (ICC), pp. 876-881, 2017.

[17] Maral G., Bousquets M., \& Zhili Z., "Satellite Communications Systems: Systems, Techniques and Technology," John Wiley, Chichester, 2010.

[18] Wang U., She C., \& Liu J., "Transmission systems, constellation inference for polar LEO satellite networks by delay probing," European Transactions on Telecommunications, vol 19, no. 3, pp. 285-297, 2008. 
[19] M. Nasseri, E. Ershadi and R. Danesfahani, "Examination and analysis of polar low earth orbit constellation," International Conference on Wireless Communications \& Signal Processing, pp. 1-5, Nanjing, 2009.

[20] Maini A.K. \& Agrawal V., "Satellite Technology-Principles and Applications," John Wiley, Chichester, 2007.

[21] Portillo I. D., Cameron B. G., \& Crawley E. F., "A Technical Comparison of Three Low Earth Orbit Satellite Constellation Systems to Provide Global Broadband," International Astronautical Congress, vol. 159, pp. 123-135, 2019.

[22] Cakaj S., Kamo B,. Lala A., Rakipi A., "The Coverage Analysis for Low Earth Orbiting Satellites at Low Elevation", International Journal of Advanced Computer Science and Applications (IJACSA), Tirana, 2014.

[23] Nejat A., "Digital Satellite Communications Systems and Technologies-Military and Civil Applications," KluwerAcademic Publishers, Boston, US, 1992

[24] Malaguti, N., "Coordination of an Earth Station with Stations of Terrestrial Services as well as Earth Stations Operating in Opposite Direction," ITU-BR, Geneva, Switzerland, 2002.

[25] Blonstein L., "Communications Satellites, The Technology of Space Communications," Heinemann Publishers, London, UK, 1987.

[26] Feher K., "Digital Communications, Satellite Earth Station Engineering," Prentice-Hall, Englewood Cliffs, New Jersey, US, 1983.

[27] Horwath J. \& Other, "Broadband Backhaul Communication for Stratospheric Platforms: the Stratospheric Optical Payload Experiment (STROPEX),” Free-Space Laser Communications VI, 6304, San Diego, US, 2006.

\section{BIOGRAPHIES OF AUTHORS}

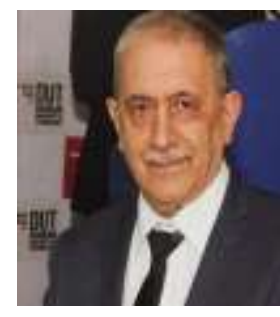

Prof. Ilcev is research leader of the Space Science Centre (SSC) for research and postgraduate studies at Durban University of Technology (DUT). He has three BSc degrees in Radio, Nautical Science and Maritime Electronics and Communications. He got MSc and $\mathrm{PhD}$ in Mobile Satellite Communications and Navigation as well. Prof. Ilcev also holds the certificates for Radio operator 1st class (Morse), for GMDSS 1st class Radio Electronic Operator and Maintainer and for Master Mariner without Limitations. He is author of several books in mobile Radio and Satellite CNS, DVB-RCS, Satellite Asset Tracking (SAT), Stratospheric Platform Systems (SCP) for maritime, land (road and railways) and aeronautical applications. 